\title{
Prevalence, Utilization and Conservation Strategies for Non-Timber Forest Products in South western Zone of Nigeria
}

\author{
S. O. Jimoh ${ }^{1}$, T. O. Amusa ${ }^{2, *}$, I. O. Azeez ${ }^{1}$ \\ ${ }^{1}$ Department of Forest Resources Management, University of Ibadan, Ibadan, Oyo State, 200001, Nigeria \\ ${ }^{2}$ Department of Forest Resources Management, University of Ilorin, Ilorin, Kwara State, 240003, Nigeria
}

\begin{abstract}
The study was carried out to investigate the prevalence and utilization of non-timber forest products (NTFPs) plant species in Omo and Shasha Forest Reserves, Southwestern Nigeria. Data were obtained through the use of structured questionnaires administered to households in forest communities. To complement information from the household survey, focus group discussions (FGDs) were also carried out in each of the sampled communities. In-depth interview (IDI) of forestry staff was further used to supplement the information collected. A simple random sampling technique was employed for the study. In Omo Forest Reserve (OFR), a total of seventy five species distributed in forty three families were recorded, while fifty six species in thirty one families were documented in Shasha Forest Reserve (SFR). Local end-uses of NTFPs include food and food supplements, snacks/sweeteners, wrapping leaves, soup and spices, beverages, dental care supplies, washing tools, roofing/construction materials, medicine and traditional rites items. While access to these resources is important to guarantee the socio-economic well being of the forest dwellers and communities, their sustainable management has not been taken seriously by policy makers thereby contributing to reduction of biodiversity and gradual but irretrievable loss of indigenous knowledge on the uses of most species. To ensure the sustainable utilization of NTFPs in these and similar tropical forest environments therefore, a number of conservation approaches are proposed.
\end{abstract}

Keywords Tropical Rainforest, Non-Timber Forest Products, Utilization, Prevalence and Conservation Strategies

\section{Introduction}

Non-timber forest products (NTFPs), sometimes referred to as minor forest products or non-wood forest products, encompass a wide range of natural resources. NTFPs are goods and services of biological origin other than wood derived from forests and allied land uses[1]. The focal view of NTFPs excludes the timber and that the products, benefits or services should come from a forest or associated ecosystems i.e. any part of plant or animal harvested for human or animal use or which contributes directly or indirectly to welfare can be described as NTFPs. These NTFPs are an integral part of the survival and development strategy for the continued well - being of man, livestock, flora, and fauna. Though both natural and plantation forests are a rich source of NTFPs, their relevance had been over shadowed in the past by the over - concentration on the timber component of the forests[2]. The timber-first orientation arose from the high premium placed on wood

* Corresponding author:

teejayui@gmail.com (T. O. Amusa)

Published online at http://journal.sapub.org/re

Copyright (C 2012 Scientific \& Academic Publishing. All Rights Reserved by early foresters as the major economic crop from forests.

The role of NTFPs in rural livelihoods is an important factor stimulating interest in bringing them back into forest management focus. Jimoh and Adebisi[2] averred that the integration of NTFPs with timber production could provide local benefits and make timber extraction more environmentally sustainable and economically viable. The relevance of NTFPs in sustainable forest management has been highlighted to include its potential in curbing environmental degradation; putting paid to insecurity of forest properties; and promoting traditional / indigenous natural resource management technique[2]. FAO[1] listed three overarching benefits of NTFPs integration in sustainable forest management as: (1) income generation for rural development; (2) more equitable sharing of the benefits of forest; and (3) local participation in forest management.

In economic terms, NTFPs contribute substantially to national economic growth and international trade. The wild plant resources contribute an income of US\$ 194-1114 per household per year in Southern Africa[3]. Globally, herbal medicines entering the international market were valued at US\$ 14 billion in 1996[4]. Jimoh and Haruna[5] reported that the contributions of NTFPs to household income around the Onigambari Forest Reserve, Nigeria, amount to $68.1 \%$ of 
total monthly income. In the Southeast of Cameroon, NTFPs have been found to contribute more than $50 \%$ to village household incomes[6]. In two out of eight villages studied in Ghana, collecting forest leaves for wrapping food, sponge-making and basket-weaving provided the main sources of income to the households[7]. In Nepal, the Forestry Department collects US\$ 15 million yearly revenue from the trading of NTFPs[8].

NTFPs occur in all the ecological zones of Nigeria and they include biotic and abiotic resources of wild species of plants and animals plus soil minerals[9]. The numbers, types, population and diversity of species which occur in the different ecological zones vary in accordance with inherent genetic characteristics, land use practices, edaphic conditions and environmental influences. The use of NTFPs in natural or planted forests is normally not restricted; hence they have been increasingly exploited without a long-term view towards their sustainable use. Equally, the rich variety of non-timber forest products in the country, many of which have been used by people for centuries, has not been well-documented. Therefore, this study was carried out to: prepare a checklist of the major non-timber yielding plant species in Omo and Shasha Forest Reserves, Southwestern Nigeria; document their utilization in the area and; proffer possible conservation approaches for sustainable utilization of these valuable products.

\section{Materials and Methods}

\subsection{The Study Areas}

The study was conducted in Omo and Shasha Forest Reserves of tropical lowland rainforest zone in southwestern Nigeria. This densely populated zone covers about $2 \%$ of the total land area of Nigeria.[10]. Within this zone, a number of studies[11, 12] have documented progressive shifts in the ecological boundaries of the tropical lowland rainforest as a result of human pressures. The cluster of forest reserves in the study area when taken together forms the Omo-Oluwa-Shasha complex (Fig. 1).

\subsection{Omo Forest Reserve}

Omo Forest Reserve (OFR) is located between Latitudes $6^{\circ} 35^{\prime}-7^{\circ} 05^{\prime} \mathrm{N}$ and Longitudes $4^{\circ} 19^{\prime}-4^{\circ} 40^{\prime} \mathrm{E}$ in the Ijebu East and North Local Government Areas of Ogun State, southwestern Nigeria. The Reserve covers an area of about 130,500 hectares forming common boundaries with Osun, Ago-Owu and Shasha Forest Reserves in Osun State and Oluwa Forest Reserve in Ondo State. The Nigerian Government legally gazetted it a forest through Order No. 10 Gazette No. 40 of 7th May 1925 which was amended in 1952[13]. The government in 1946 established a 460 ha Strict Nature Reserve (SNR) within Omo Forest Reserve. This was upgraded to a Biosphere Reserve (BR) in 1977 by UNESCO[14, 15]. The rainy season in OFR usually commences in March. The mean annual rainfall in the area ranges from about 1600 to $2000 \mathrm{~mm}$ with two annual peaks in June and September. Temperature ranges from $32.15^{\circ} \mathrm{C}$ to $21.40^{\circ} \mathrm{C}$ and a minimum relative humidity of $76.34 \%[16]$. The vegetation of the Reserve is a mixed moist semi-deciduous rainforest. Most of the forests are disturbed with a substantial part converted to monoculture plantations of Gmelina arborea in a programme assisted by loans from the World Bank and the African Development Bank to provide material for a pulp mill at Iwopin. For effective management, the reserve was subdivided into four areas or sectors viz: J1, J3, J4 and J6. These subdivisions were apportioned to enclave dwellers in isolated villages or camps. Estimated total population in the area is between 20,000 and 25,000. Farming, fishing, hunting and NTFPs gathering are the predominant occupations for the majority of the enclaves' population.

\subsection{Shasha Forest Reserve}

Shasha Forest Reserve (SFR) is located between Latitudes $7^{0} 00^{\prime}-7^{0} 30^{\prime} \mathrm{N}$ and Longitudes $4^{0} 00^{\prime}-5^{0} \mathrm{E}$ in Osun State, southwestern Nigeria. The reserve was first gazetted in 1925 as part of the Old Shasha Forest Reserve under an agreement with the Ijebu Native Authority. The reserve shares boundaries with Omo Forest Reserve on the west. The northern and eastern boundaries are with Ife Native Authority Reserve and Oluwa Forest Reserve in Osun and Ondo States respectively. The total area of the Reserve is currently 23,064 ha. Out of this, about 1,523 ha are under plantation of various species such as Gmelina arborea, Tectona grandis, Terminalia spp, Pinus spp and Nauclea diderichii. The remaining $21,541 \mathrm{ha}$ is currently dominated by pockets of degraded natural forests characterized with broken canopy[17]. The rainy season in SFR usually commences from March/April and lasts till November. Total Annual rainfall ranges from $887 \mathrm{~mm}$ to $2,180 \mathrm{~mm}$. The mean annual temperature is about $26.5^{\circ} \mathrm{C}$ with annual range of between $19.5^{\circ} \mathrm{C}$ and $32.5^{\circ} \mathrm{C}$. The original vegetation structure of Shasha Forest Reserve is three storied with scattered emergents[18]. The Reserve is subdivided into two major areas viz, Areas 4 and 5. There are about forty communities within and around the Forest Reserve. The population of these communities range from 200 to 2000 inhabitants.

\subsection{Data Collection and Sampling Procedure}

Data were collected through socio-economic approach. This included household survey; focus group discussion; and in-depth interview with forest communities and forestry personnel. Four enclaves were sampled in the J4 Sector of Omo Forest Reserve, and six communities within and around Shasha Forest Reserve. Household surveys were conducted among randomly selected sub-set of households in each site. In a sampling intensity (S.I), 30\% S.I was used where total number of households was less than 100 while $10 \%$ S.I was used where number exceeded 100[19]. Thus, a total of 81 and 85 households were sampled in OFR and SFR 
respectively. To complement information from the household survey, focus group discussions (FGDs) were carried out in each of the sampled communities. This provided forum for weighing the relative importance of identified NTFPs. Semi-structured questionnaires with a mixture of open-ended and fixed-response questions through an in-depth interview were further used to access information from the forestry staff in the two reserves. There were fourteen Uniform and Technical forestry personnel in OFR, while SFR had twenty as at the time of the survey. All the Uniform and Technical forestry personnel (fourteen) in OFR participated in the survey, while twelve questionnaires were administered in SFR (being the number of those available and willing to participate in the survey).

\subsection{Data Analysis}

The Chi-square test- a nonparametric test which involves the analysis of the frequency or percentage count of two different variables and hence the relationship between these variables was used to analyse information gathered on the utilization of NTFPs in the study area. The data analyses were carried out using the Statistical Package for Social Sciences[20].

\section{Results and Discussion}

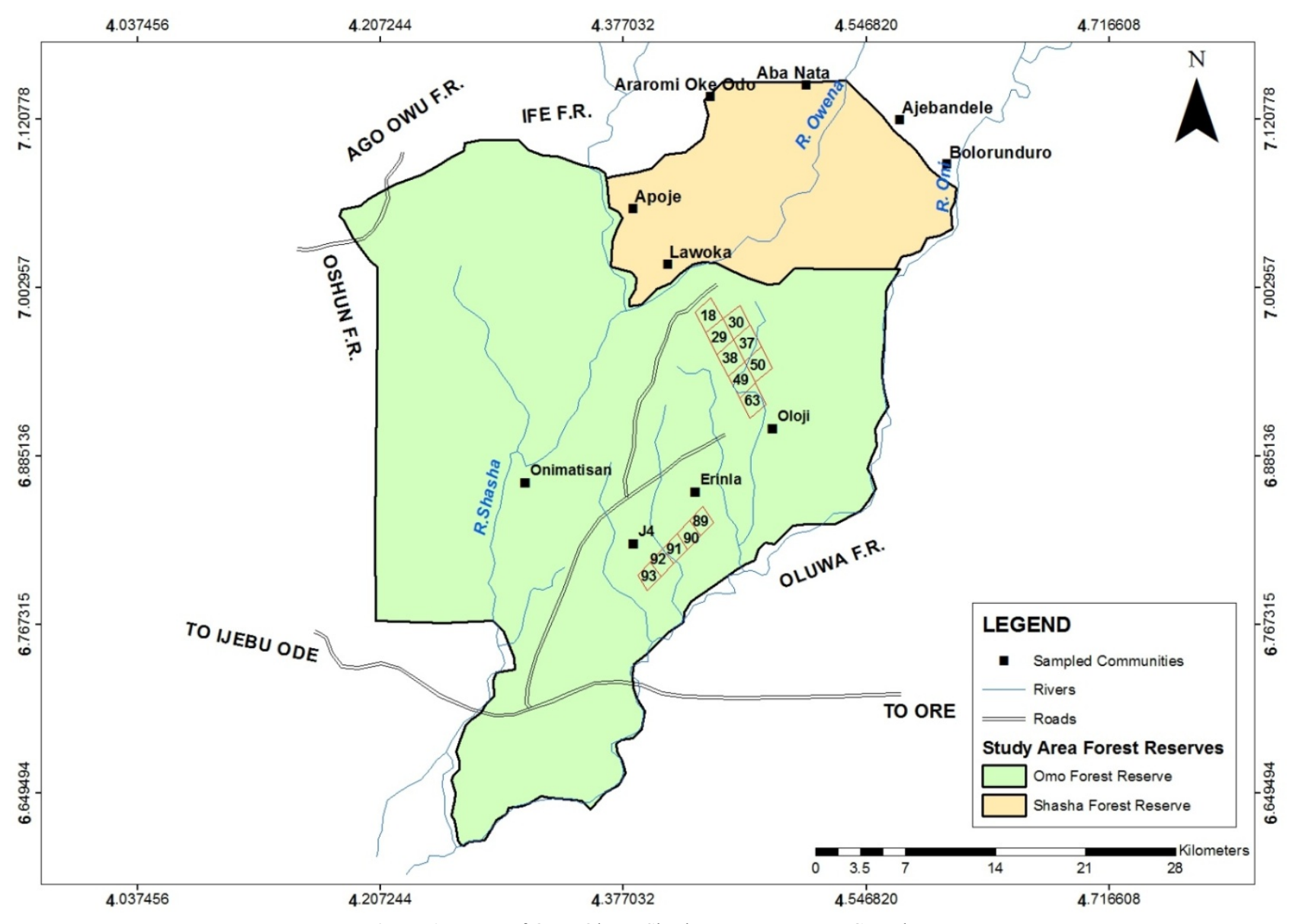

Figure 1. Map of Omo-Oluwa-Shasha Forest Reserves Complex

\subsection{Prevalence and Uses of NTFPs in the Study Area}

Seventy five NTFP species distributed in forty three families were recorded in OFR, while fifty six species which spread across thirty one families were documented in SFR (Table 1). Twenty nine species were peculiar to OFR including; Epo omo (bark of Cordia millenii), Ewe ogbo (leaf of Parquetina nigrescens) Gbokonisa (Beilschimiedia mannii) and Gbongbo agboin (root of Piptedeniastrum africanum), among others. On the other hand, nine species were peculiar to SFR including: Asunwo (Senna alata), Ayin (Annogeissus leiocarpa), Dongoyaro (Azadirachta indica), Opepe (Nauclea diderichii), Ewuro (Vernonia amygdalina), Igba (Parkia biglobosa), Iya (Daniellia oliveri), Ose (Adansonia digitata), Osun (Pterocarpus osun), and Sanda (Carpolobia spp). Local end-uses of NTFPs in the two reserves include food and food supplements, snacks/sweeteners, wrapping leaves, soup/soup ingredients, beverages, chew-stick, washing items, roofing/construction, medicine and traditional rites. Medicinal uses occupy the modal use category in both sites (51 .47\%; Fig. 2). NTFPs are extracted from different plant life-forms including trees, shrubs, herbs, palms and lianas with trees constituting the highest (Fig. 3). Virtually all parts of the plants except the flower find usefulness as NTFPs. Leaves (30.44\%), fruits $(25.96 \%)$ and bark $(16.19 \%)$ are the predominant parts commonly utilized as NTFPs in both sites (Table 2). 
Table 1. Prevalence and Uses of NTFPs in Omo and Shasha Forest Reserves, Nigeria

\begin{tabular}{|c|c|c|c|c|c|}
\hline $\begin{array}{l}\text { Utilization } \\
\text { Category }\end{array}$ & Species & Local Name & Habit & Site & Part(s) used \\
\hline \multirow{2}{*}{$\begin{array}{l}\text { Species Used as } \\
\text { Food/Main Dish }\end{array}$} & Treculia africana Decne. (Moraceae) & Afon & Tree & OFR, SFR & Fruit, Seed \\
\hline & Artocarpus altilis (Parkinson) Fosberg (Moraceae) & Berefuutu & Tree & OFR, SFR & Fruit \\
\hline $\begin{array}{l}\text { Species Used as } \\
\text { Food supplement }\end{array}$ & Edible mushrooms (Varies) & Ataase (Olu) & Fungi & OFR, SFR & Whole fungus \\
\hline \multirow{9}{*}{$\begin{array}{c}\text { Species Used as } \\
\text { Snacks/Sweetener } \\
\text { s }\end{array}$} & Treculia africana Decne. (Moraceae & Afon & Tree & OFR, SFR & Fruit, Seed \\
\hline & Chrysophylum albidum, G. Don (Sapotaceae) & Agbalumo & Tree & OFR, SFR & Fruit \\
\hline & $\begin{array}{c}\text { Synsepalum dulcificum (Schumach. \& Thonn.) } \\
\text { Daniell (Sapotaceae) }\end{array}$ & Agbayun & Shrub & OFR, SFR & Fruit \\
\hline & $\begin{array}{c}\text { Tetracarpidium conophorum (Mull.Arg.) Hutch.\& } \\
\text { Dalziel (Euphorbiaceae) }\end{array}$ & Asala & $\begin{array}{l}\text { Climbing } \\
\text { shrub }\end{array}$ & OFR, SFR & Fruit \\
\hline & Blighia sapida, K.D.Koenig (Sapindaceae) & Isin & Tree & OFR & Fruit \\
\hline & $\begin{array}{l}\text { Irvingia gabonensis (Aubry-Lecomte ex O'Rorke) } \\
\text { Baill (Irvingiaceae) }\end{array}$ & Ooro & Tree & OFR & Fruit \\
\hline & Garcinia kola, Heckel. (Clusiaceae) & Orogbo & Tree & OFR, SFR & Seed \\
\hline & Sida veronicifolia, Lam. (Malvaceae) & Esi-ile & Creeper & OFR & Fruit \\
\hline & Dialium guineense Willd. (Cealsalpinaceae) & Omoyin & Tree & OFR & Fruit \\
\hline \multirow{23}{*}{$\begin{array}{l}\text { Species Used as } \\
\text { Soup/Soup } \\
\text { ingredients/ } \\
\text { Spices/Condimen } \\
\text { ts }\end{array}$} & $\begin{array}{c}\text { Irvingia gabonensis (Aubry-Lecomte ex O'Rorke) } \\
\text { Baill (Irvingiaceae) }\end{array}$ & Aapon (Ogbono) & Tree & OFR, SFR & Seed \\
\hline & Brachystegia eurycoma Harms, B. (Caesalpiniaceae) & Akporachi (EKU) & Tree & OFR & Seed \\
\hline & Afzelia africana Sm. (Caesalpiniaceae) & Apa & Tree & OFR, SFR & Seed, \\
\hline & $\begin{array}{c}\text { Crassocephalum crepidioides (Benth.) S. Moore } \\
\text { (Asteraceae) }\end{array}$ & Ebolo & Herb & OFR, SFR & Leaf \\
\hline & Ceiba pentandra, (L.) Gaertn. (Malvaceae) & Eegun & Tree & OFR & Leaf \\
\hline & Adenopus breviflorus Benth. (Cucurbitaceae) & Egusi ile & Climber & SFR & Leaf \\
\hline & Boerhavia diffusa, L. (Nyctaginaceae) & Etiponnla & Herb & OFR & Leaf \\
\hline & Vernonia amygdalina, Del. (Asteraceae) & Ewuro & $\begin{array}{l}\text { Forb or } \\
\text { Shrub }\end{array}$ & SFR & Leaf \\
\hline & $\begin{array}{l}\text { Beilschimiedia mannii, (Meisn.) Benth. \& Hook. f } \\
\text { (Lauraceae) }\end{array}$ & Gbokonisa & Tree & OFR & Leaf \\
\hline & Erythrina senegalensis D.C (Fabaceae) & Ilaka ile & Tree & OFR & Leaf \\
\hline & Mondia whitei (Hook.f.) Skeels (Asclepiadaceae) & Isigun & Herb & OFR & Root \\
\hline & Gongronema latifolium Benth. (Asclepiadaceae) & Iteji & $\begin{array}{l}\text { Shrub or } \\
\text { Tree }\end{array}$ & OFR, SFR & Leaf \\
\hline & Cissampelos owariensis, P. Beau (Menispermaceae) & Jenjoko & Climber & OFR & Leaf \\
\hline & Dichapetalum pallidum (Dichapetalaceae) & Marigbo & Tree & OFR & Leaf \\
\hline & Adansonia digitata, L. (Malvaceae) & Ose & Tree & SFR & Leaf \\
\hline & Pterocarpus osun Craib. (Papilionaceae) & Osun & Tree & SFR & Leaf \\
\hline & Peperomia pellucida, (Linn.) HB \& K. (Piperaceae) & Rinrin & Herb & SFR & Leaf \\
\hline & Capsicum spp. (Solanaceae) & Ata ijosi & Shrub & OFR, SFR & Fruit \\
\hline & $\begin{array}{c}\text { Aframomum sceptrum (Oliv. \& Hanb.) K Schum. } \\
\text { (Zingiberaceae) }\end{array}$ & Ata oguro & Herb & OFR, SFR & Fruit, Seed \\
\hline & Zingiber officinale, Roscoe (Zingiberaceae) & Ata-ile (Ginger) & Tuber & OFR & Root \\
\hline & $\begin{array}{c}\text { Zanthoxylum zanthoxyloides, (Lam.) Zepern. \& } \\
\text { Timler (Rutaceae) }\end{array}$ & Igi ata & Shrub & OFR, SFR & Leaf \\
\hline & Piper guineense Schumach. \& Thonn. (Piperaceae) & Iyere & Liana & OFR, SFR & $\begin{array}{l}\text { Fruit, Seed, } \\
\text { Leaf }\end{array}$ \\
\hline & $\begin{array}{c}\text { Parkia biglobossa, (Jacq.) R.Br. ex G.Don. } \\
\text { (Mimosaceae) }\end{array}$ & Igba & Tree & SFR & Seed \\
\hline $\begin{array}{l}\text { Species Used as } \\
\text { Beverage }\end{array}$ & Raphia hookeri G.Mann \& H.Wendl. (Palmae) & Ako (Raffia palm) & Palm & OFR & Stem \\
\hline \multirow{2}{*}{$\begin{array}{l}\text { Species Used as } \\
\text { Chew-stick }\end{array}$} & $\begin{array}{c}\text { Zanthoxylum zanthoxyloides, (Lam.) Zepern. \& } \\
\text { Timler (Rutaceae) }\end{array}$ & Igi ata & Shrub & OFR, SFR & Root \\
\hline & Massularia acuminata (G Don) Bullock (Rubiaceae) & $\begin{array}{l}\text { Pako ijebu (chew } \\
\text { stick) }\end{array}$ & Tree & OFR, SFR & Stem, Branch \\
\hline \multirow{2}{*}{$\begin{array}{l}\text { Species Used for } \\
\text { Packaging }\end{array}$} & Mitragyna ciliata, Aubrév. \& Pellegr (Rubiaceae) & Abura & Tree & OFR, SFR & Leaf \\
\hline & $\begin{array}{l}\text { Thaumatococcus daniellii (Benn.) Benth. } \\
\text { (Marantaceae) }\end{array}$ & Ewe eeran & Herb & OFR, SFR & Leaf \\
\hline $\begin{array}{l}\text { Species Used for } \\
\text { Washing }\end{array}$ & Momordica angusticephalas, Harms (Cucurbitaceae) & Kainkanin & Climber & OFR, SFR & Fruit \\
\hline \multirow{6}{*}{$\begin{array}{l}\text { Species Used for } \\
\text { Medicines }\end{array}$} & Parinari excelsa Sabine (Chrysobalanaceae) & Abere & Tree & OFR, SFR & Fruit, Bark \\
\hline & Mitragyna ciliata, Aubrév. \& Pellegr (Rubiaceae) & Abura & Tree & OFR, SFR & Leaf, Bark \\
\hline & Terminalia ivorensis A. Chev. (Combretaceae) & Afara dudu & Tree & OFR, SFR & Bark \\
\hline & Treculia africana Decne. (Moraceae) & Afon & Tree & OFR, SFR & Fruit, Seed \\
\hline & Musanga cecropioides R.Br. (Moraceae) & Aga & Tree & OFR, SFR & Stem, Branch \\
\hline & $\begin{array}{l}\text { Gossweilerodendron balsamiferum (Verm.) Harms } \\
\text { (Caesalpiniaceae) }\end{array}$ & Agba & Tree & OFR, SFR & Bark \\
\hline
\end{tabular}




\begin{tabular}{|c|c|c|c|c|c|}
\hline & $\begin{array}{c}\text { Piptadeniastrum africanum, (Hook.f.) Brenan } \\
\text { (Mimosaceae) }\end{array}$ & Agboin & Tree & OFR & Root \\
\hline & Culcasia saxatilis, A.Chev. (Lamiaceae) & Agunmona & Herb & OFR & Fruit \\
\hline & $\begin{array}{l}\text { Tetrapleura tetraptera (Schumach. \& Thonn.) Taub. } \\
\text { (Mimosaceae) }\end{array}$ & Aidan & Tree & OFR, SFR & Fruit \\
\hline & $\begin{array}{c}\text { Newbouldia laevis (P. Beauv.) Seem. ex Bureau } \\
\text { (Bignoniaceae) }\end{array}$ & Akoko & $\begin{array}{l}\text { Tree or } \\
\text { Shrub }\end{array}$ & OFR, SFR & Leaf \\
\hline & Monodora myristica (Gaertn) Dunal. (Annonacae) & Ariwo & Tree & OFR, SFR & Seed, Bark \\
\hline & Rauvolfia vomitoria Afzel. (Apocynaceae) & Asofeyeje & Tree & OFR, SFR & Leaf \\
\hline & Senna alata (L.) Roxb. (Caesalpinaceae) & Asunwo & Shrub & SFR & Leaf \\
\hline & Aframomum melegueta K. Schum. (Zingiberaceae) & Ataare & Herb & OFR, SFR & Fruit, Seed \\
\hline & Pativera alliaceae, L. (Phytolaccacea) & Awogba & Herb & OFR & Leaf \\
\hline & $\begin{array}{c}\text { Annickia (syn. Enantia) chlorantha (Oliv.) Setten \& } \\
\text { Maas (Annonaceae) }\end{array}$ & Awopa (Yaani) & Tree & OFR, SFR & Bark \\
\hline & Alstonia boonei De Wild. (Apocynaceae) & Awun & Tree & OFR, SFR & Bark \\
\hline & $\begin{array}{l}\text { Anogeissus leiocarpus (DC.) Guill. \& Perr. } \\
\text { (Combretaceae) }\end{array}$ & Ayin & Tree & SFR & Fruit \\
\hline & Allium sativum, L. (Alliaceae) & Ayuu (Garlic) & Creeper & OFR & Fruit \\
\hline & Azadirachta indica, , L. (Meliaceae) & Dongoyaro & Tree & SFR & Bark \\
\hline & Ceiba pentandra, (L.) Gaertn. (Malvaceae) & Eegun & Tree & OFR & Leaf \\
\hline & Chloris pilosa Schumach. (Poaceae) & Eeran & Grass & OFR & Leaf \\
\hline & Hunteria umbellata (K. Schum) (Apocynaceae) & Eerin & Tree & OFR, SFR & Fruit \\
\hline & $\begin{array}{c}\text { Nauclea diderrichii (De Wild. \& T.Durand) Merrill } \\
\text { (Rubiaceae) }\end{array}$ & Egbesi & Shrub & OFR, SFR & Leaf \\
\hline & Adenopus breviflorus Benth. (Cucurbitaceae) & Egusi ile & Climber & SFR & Leaf, Bark \\
\hline & Momordica foetida Schumach. (Cucurbitaceae) & Ejinrin & Herb & OFR, SFR & Leaf \\
\hline & Picralima nitida (Stapf) Th. \& H. Dur (Apocynaceae) & Erin & Tree & OFR, SFR & Seed, Bark \\
\hline & Cnestis ferruginea, DC (Connaraceae) & Gboyin-gboyin & Tree & OFR & Leaf \\
\hline & Ocimum basilicum L. (Labiatae) & Igi ota & Shrub & OFR, SFR & Stem, Branch \\
\hline & Ageratum conyzoides, L. (Asteraceae) & Imi esu & Herb & OFR, SFR & Leaf \\
\hline & Ficus exasperata Vahl (Moraceae) & Ipin & Tree & OFR, SFR & Leaf \\
\hline & Blighia sapida, K.D.Koenig (Sapindaceae) & Isin & Tree & OFR & $\begin{array}{l}\text { Fruit, Leaf, } \\
\text { Bark }\end{array}$ \\
\hline & $\begin{array}{l}\text { Daniellia oliveri (Rolfe) Hutch. \& Dalz. } \\
\text { (Caesalpinoceae) }\end{array}$ & Iya & Tree & SFR & Root \\
\hline & Spondias mombin L. (Anacardiaceae) & Iyeye & Tree & OFR, SFR & Fruit, Leaf \\
\hline & Cissampelos owariensis, P. Beau (Menispermaceae) & Jenjoko & Climber & OFR & Leaf, Bark \\
\hline & Buchholzia coriacea Engl. (Capparaceae) & $\begin{array}{l}\text { Kokoroijemu/Obii } \\
\text { koro (wonderful } \\
\text { kola) }\end{array}$ & Tree & OFR, SFR & Seed \\
\hline & Trichilia rubescens, Oliv (Meliaceae) & Kurere & Tree & OFR & Stem, Branch \\
\hline & Hippocratea indica Willd. (Celastraceae) & Mawole & $\begin{array}{c}\text { Climbing } \\
\text { shrub or } \\
\text { Liana }\end{array}$ & OFR, SFR & Root \\
\hline & Khaya ivorensis A. Chev. (Meliaceae) & Oganwo & Tree & OFR, SFR & Bark \\
\hline & $\begin{array}{c}\text { Parquetina nigrescens (Afzel.) Bullock } \\
\text { (Asclepiadaceae) }\end{array}$ & Ogbo & Twine & OFR & Leaf \\
\hline & Sansevieria trifasciata, Prain. (Ruscaceae-Agavaceae) & Oja ikoko & Creeper & OFR & Leaf \\
\hline & Abrus precatorius, L. (Fabaceae) & Oju ologbo & Tree & OFR & Seed \\
\hline & Erigeron floribundus (Kunth) Sch.Bip. (Asteraceae) & Olowojeja & Herb & OFR & Leaf \\
\hline & Cordia millenii, Baker (Boraginaceae) & Omo & Tree & OFR & Bark \\
\hline & Gardenia erubescens, Stapf \& Hutch. (Rubiaceae) & Oruwon & $\begin{array}{l}\text { Shrub or } \\
\text { Tree }\end{array}$ & OFR & Leaf \\
\hline & Morinda lucida Benth. (Rubiaceae) & Oruwo & Tree & OFR, SFR & Leaf, Bark \\
\hline & Adansonia digitata, L. (Malvaceae) & Ose & Tree & SFR & $\begin{array}{l}\text { Root, Bark, } \\
\text { leaf }\end{array}$ \\
\hline & Lophira alata Banks ex Gaertn. (Ochnaceae) & Pahan & Tree & OFR, SFR & Bark \\
\hline & Kigelia africana (Lam.) Benth. (Bignoniaceae) & Pandoro & Tree & OFR, SFR & Fruit \\
\hline & Raphia hookeri G.Mann \& H.Wendl. (Palmae) & Ako (Raffia palm) & Palm & OFR & Leaf \\
\hline Species Used for & Bambusa vulgaris Schrad. (Poaceae) & Oparun & Grass & OFR, SFR & Stem, Leaf \\
\hline & Morinda lucida Benth. (Rubiaceae) & Oruwo & Tree & OFR, SFR & Bark \\
\hline & Parinari excelsa Sabine (Chrysobalanaceae) & Abere & Tree & OFR, SFR & Fruit \\
\hline $\begin{array}{l}\text { Species Used for } \\
\text { Traditional rites }\end{array}$ & $\begin{array}{c}\text { Newbouldia laevis (P. Beauv.) Seem. ex Bureau } \\
\text { (Bignoniaceae) }\end{array}$ & Akoko & $\begin{array}{l}\text { Tree or } \\
\text { Shrub }\end{array}$ & OFR, SFR & Leaf \\
\hline & $\begin{array}{c}\text { Cola acuminata Schott \& Endl. (Malvaceae- } \\
\text { Sterculioideae) }\end{array}$ & Obi Abalaye & Tree & OFR, SFR & Fruit \\
\hline Species Used as & Afzelia africana Sm. (Caesalpiniaceae) & Apa & Tree & OFR, SFR & Leaf \\
\hline $\begin{array}{l}\text { Animal feed and } \\
\text { Care }\end{array}$ & Euphorbia hirta L (Euphorbiacae) & Tomide & Herb & OFR & Leaf \\
\hline
\end{tabular}




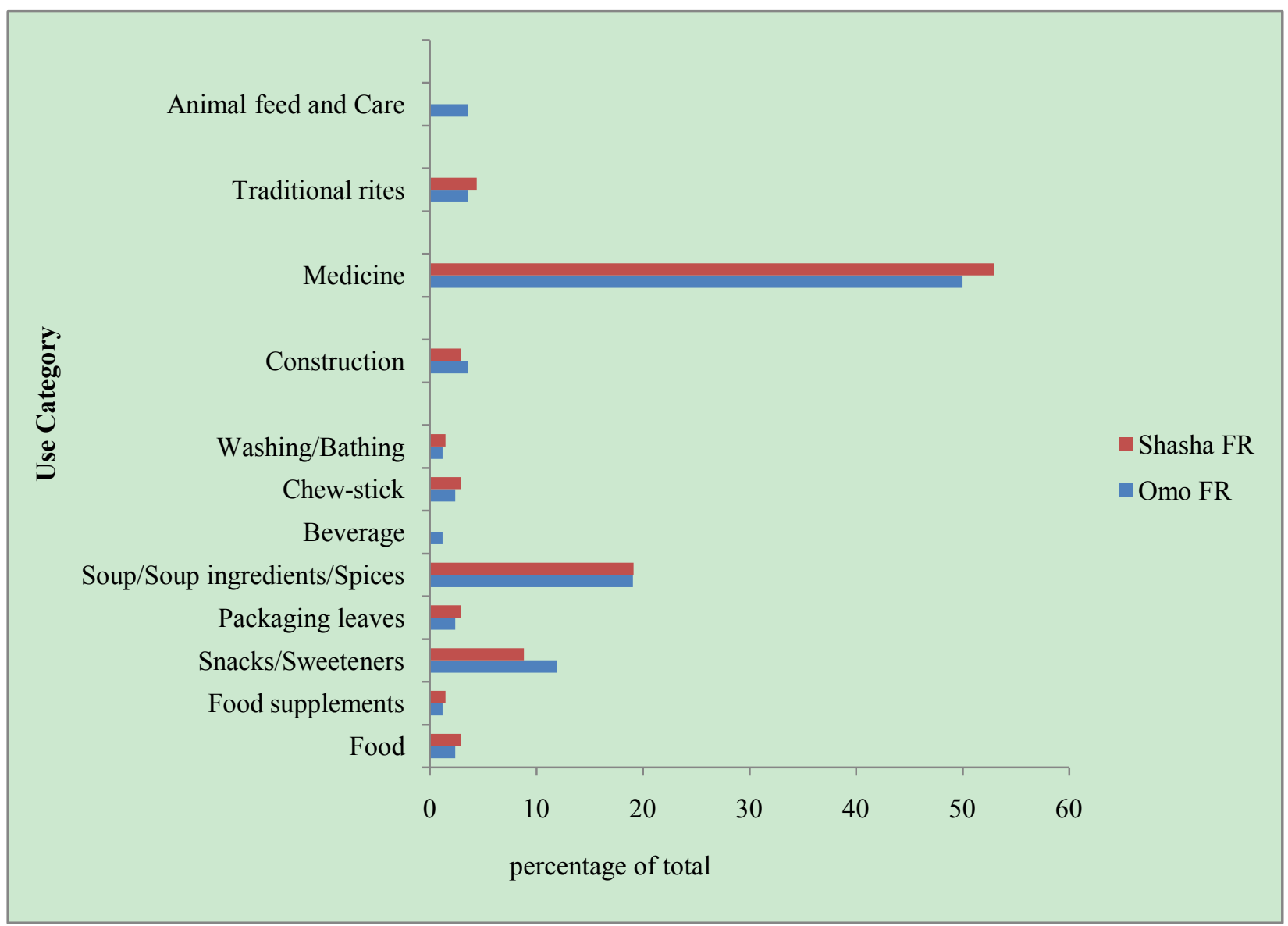

Figure 2. Utilization Categories of NTFPs in Omo and Shasha Forest Reserves Expressed in Percentage

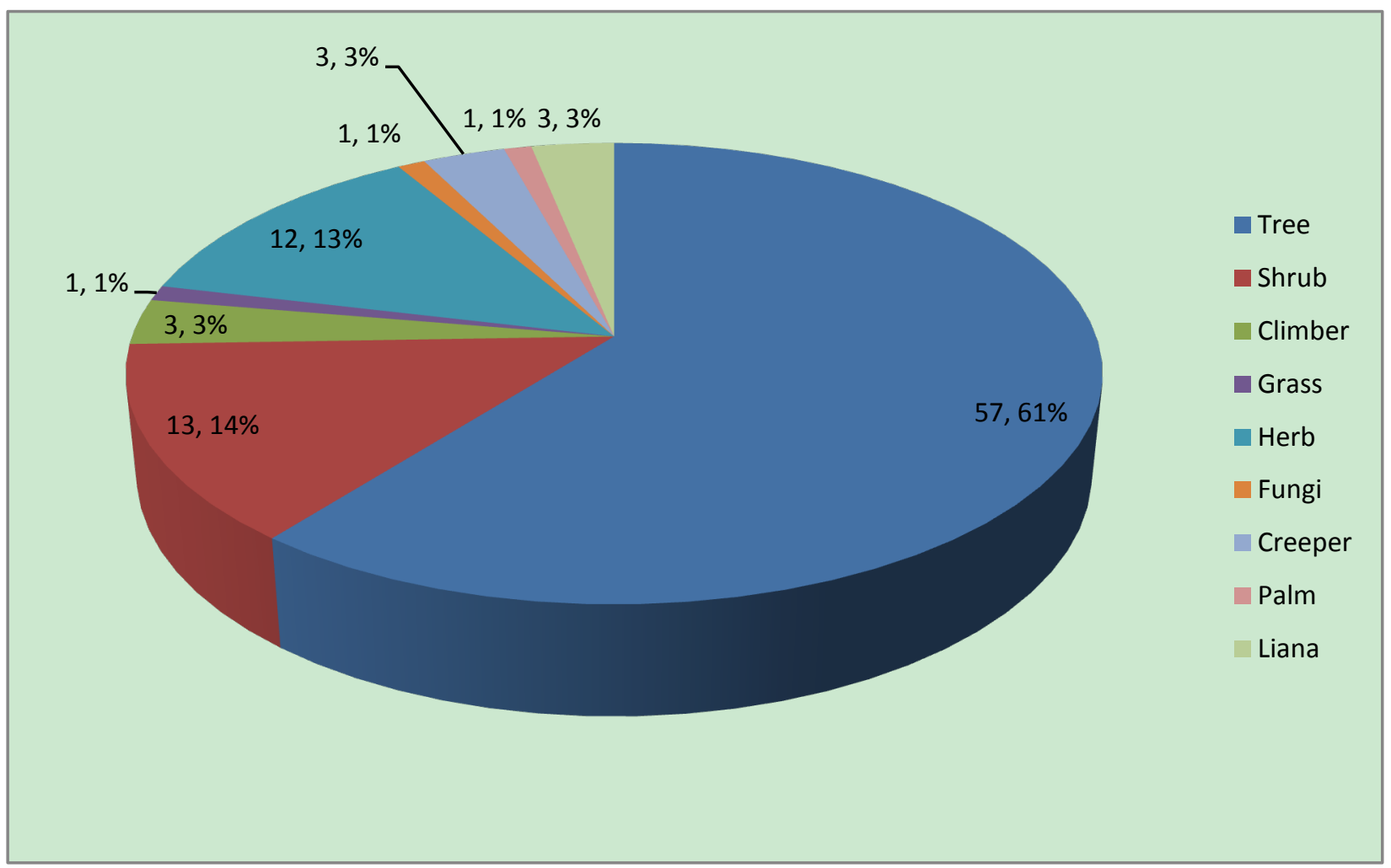

Figure 3. NTFPs Life-form and Corresponding Number of Plant Species Expressed in Percentage 
Table 2. Summary of NTFPs Parts and Corresponding Number of Plant Species

\begin{tabular}{|c|c|c|c|c|}
\hline \multirow{2}{*}{} & \multicolumn{2}{|c|}{ Number of Species } & \multicolumn{2}{c|}{ Percentage of Total } \\
\cline { 2 - 5 } & Omo FR & $\begin{array}{c}\text { Shasha } \\
\text { FR }\end{array}$ & Omo FR & $\begin{array}{c}\text { Shasha } \\
\text { FR }\end{array}$ \\
\hline Fruit & 22 & 20 & 22.92 & 28.99 \\
\hline Seed & 12 & 07 & 12.50 & 10.14 \\
\hline Flower & 0 & 0 & 0.00 & 0.00 \\
\hline Stem/Branch/Latex & 12 & 05 & 22.92 & 7.25 \\
\hline Leave & 32 & 19 & 33.33 & 27.54 \\
\hline Root & 5 & 05 & 5.21 & 7.25 \\
\hline Bark & 13 & 13 & 13.54 & 18.84 \\
\hline
\end{tabular}

\subsection{NTFPs for Medicinal Purposes}

Forty two medicinal plant species were documented for OFR while thirty six were recorded for SFR. Medicinal plant species of great local importance in the study area include: Piptedeniastrum africanum, Tetrapleura tetraptera, Annickia (syn. Enantia) chlorantha, Bucholzia coriaceae, Hippocratea indica, Piper guineense, Morinda lucida, and Kigelia africana. Several factors account for dependency of the communities on traditional medicine. These include: culture, efficacy, holistic nature of plant therapy, cost, availability, accessibility, poverty, and poor infrastructure[21]. The knowledge of medicinal uses of these plants is transferred from generation to generation.

\subsection{NTFPs for Culinary Uses}

The culinary uses of NTFPs include their consumption as food, food supplements, snacks, spices, condiments, flavouring and beverages. In this category, thirty two and twenty four plant species were documented for OFR and SFR respectively. Important NTFPs used as forest food and food supplement include: Treculia africana, Artocarpus artilis and mushroom. Similarly, the leaves and seeds of some plant species are used as vegetables, soup/soup ingredients and spices. This is the case with such species as Brachystegia eurycoma, Capsicum spp, Crassocephalum crepidioides, Beilschimiedia mannii, Irvingia gabonensis, and Piper guineense. Wild fruits, which contain vital nutrients (carbohydrates, protein, and minerals) and essential vitamins are also important in the study area, especially for growing children, who are prone to malnutrition and related diseases[22]. Among the indigenous fruits with considerable commercial potential in the study area are: Irvingia spp., Synsepalum dulcificum, Tetracarpidium conophorum, Garcinia cola, Chrysophylum albidum, and Dialium guineense.

\subsection{NTFPs for Miscellaneous Functions}

Two plant species, Zanthoxylum zanthoxyloides and Massularia acuminata are commonly used as chew-stick (for tooth care) in the study area. Momordica angusticephalas is also used as local sponge for washing and/or bathing. Bambusa vulgaris, Morinda lucida and Raphia hookeri are widely used for construction purposes. Parinari excelsa, Newbouldia laevis and Cola acuminata are important NTFPs for social, cultural and religious functions. In the same vein, Euphorbia hirta, Afzelia africana and Carpolobia lutea are employed in animal feed and care. Euphorbia hirta is fed to rabbits, the leaves of Afzelia africana are lopped as cattle forage while, Carpolobia lutea, which is also widely exploited from the forest reserves is particularly used for driving cattle by Fulani herdsmen.

Ola-Adams[23] recorded 54 wild or semi-wild relatives of cultivated crops which also abound in the Omo Forest Reserve. Such species include species of Capsicum, Coffea, Cola, Dioscorea, Solanum, Irvingia and Ipomea. Similarly, Jimoh[21] listed 35 NTFPs of high priority attention in Shasha Forest Reserve. Some of these include: Irvingia gabonensis, Chrysophylum albidum, Anninckia (syn. Enantia) chlorantha and Piper guineense. Etkin and Ross[24] have also documented about 119 plants as foods in the savannah ecozone of Nigeria. Similarly, Okafor et al[25] identified 8 NTFPs from the mangrove swamp, 19 traded products from the moist forest, 17 from the southern guinea savannah, 12 in the Sudan savannah and 56 for the whole country.

According to Okafor et al.,[25], forest foods which are either eaten raw or processed form the major intake of proteins, vitamins, minerals, fats and carbohydrates among the majority of rural communities in Nigeria. Many NTFPs provide both social and economic benefits to rural communities. Some of these commodities also play an economic role in the national and regional economies. Extraction of NTFPs for local consumption and/or markets is carried out as part of livelihood strategies at household level. Arnold[26] reported NTFP species are found cultivated in gardens and farms for ease of access, control and management.

For the time being, while there is a full Department of Non-Timber Forest Products in the Ogun State Ministry of Forestry which holds the jurisdiction over the Omo Forest Reserve based on the development of NTFPs in non-reserve areas within the State, NTFPs operation is still a sub-sector under the Department of Forest Management in the Osun State Ministry of Environment. Based on this, the development of NTFPs in Osun State remained neglected and relegated to harvesters' payment of a token fee to register with Forest Management Unit (FMU) Authorities. Generally, NTFPs are still being regarded as minor forest products and they are not subjects of GDP calculations hence they are treated with levity.

\subsection{Conservation Approaches for Sustainable Utilization of NTFPs}

Several approaches have been suggested for the conservation of NTFPs. These include; research intervention (including regular and appropriate inventory), domestication of selected species; in-situ and ex-situ conservation; conservation through biotechnology; NTFPs trade regulation; medicinal plants farming; legal and institutional reforms among others[27, 28, 2]. The following strategies as 
expounded by Subedi[29] are further examined:

\subsection{Improving Production from the Wild on a Sustainable Basis}

This will involve the management of natural regeneration of NTFP species and will not deteriorate the resource base in the condition where the extraction of resources does not exceed the production capacity of the resource base. Controlled harvesting technique will be a simple, easy and inexpensive management system to implement by community forest user groups (CFUGs) for natural regeneration of NTFPs in forest land. This option will ensure the natural regeneration in the controlled area and check the over exploitation of NTFPs. Controlled harvesting can be enforced by applying one or more of the following alternatives: rotational harvesting, fixing of harvesting regimes and selective harvesting. These will include: cleaning the site, providing light, space, etc on the forest land. Other management applications such as thinning, coppicing, etc. as needed, will also be maintained to ensure high yields. This strategy will be useful for high quality and high production of species. However, it will be costly and quite tedious.

\subsection{Domestication}

The domestication of promising, under-exploited species in private farm land, community forest land and leasehold forest land can contribute a lot in this sector. Some important stages among them are characterization, germplasm exploration, vegetative propagation, genetic selection, and incorporation into a sustainable land-use system. For more important NTFP species, strategies for more intensive and systematic domestication might include[30]:

- screening of candidate species through species trials on farmland in collaboration with farm households;

- identification of preferred characteristics of chosen species;

- seed collection and distribution;

- study of interactions between genotype and environment; and

- establishment of seed orchard.

\subsection{Conservation Education and Training Activities}

Providing conservation education in conjunction with technical assistance may be a way to reach forest users in order to help them see their resource situation from a different perspective. Similarly, there is a need to provide training and conservation educational activities on NTFP for extension workers and forest users.

\subsection{Local Value Addition}

NTFPs collected from forest or cultivated land can be sold in different forms - crude raw materials, improved raw materials or processed raw materials. This can also be through different market channels. An improved market channel could bring out more value than a primitive one. Value addition can also make the same products more valuable so that it plays a greater role in the local economy. When value additions are encouraged for NTFPs, it will create employment for local people and help to uplift their living standard. This will further motivate the local communities to help ensure conservation of NTFP species.

\section{Recommendation}

NTFPs are a very crucial component of forest ecosystem which deserves better attention of policy makers and other forest stakeholders, hence informed policy interventions, equity of access and use, and sustainable harvesting of the multitude of resources is essential for both economic development and sustainable forest management.

\section{REFERENCES}

[1] FAO, Resource assessment of non-wood forest products: Experience and biometric principles (eds., Jennifer, L.G., Kirsti, T and Nell, B.). FAO, Rome 2001. 109Pp

[2] S.O. Jimoh, and L.A. Adebisi, Non-timber forest products and sustainable forest management in Nigeria. In: Sustainable Forest Management in Nigeria: Lessons and Prospects (eds., L. Popoola, P. Mfon and P.I. Oni). Proceedings of the $30^{\text {th }}$ Annual Conference of the Forestry Association of Nigeria, held in Kaduna, Kaduna State.7-11 ${ }^{\text {th }}$ November, 2005. Pp 266-277

[3] S. Shackleton,.; C. Shackleton,. and B. Cousins,. Re-valuing the communal lands of Southern Africa: New understanding of rural livelihoods 2000. ODI Natural Resource Perspectives no. 62. The Overseas Development Institute, London

[4] Secretariat for Convention on Biological Diversity SCBD, Sustainable management of non-timber forest resources 2001. CBD Technical series 6. Secretariat of the Convention on Biological Diversity, Montreal, Canada

[5] S.O. Jimoh, and E. A. Haruna, Contributions of Non-Timber Forest Products to Household food security and income around Onigambari forest reserve, Oyo State, Nigeria. Journal of Environmental Extension. Vol 6. 2007. Pp 28-33

[6] IUCN, Evaluation of the contribution of forests to household incomes and policy implications. Unpublished report. IUCN, Yaoundé, Cameroon un dated

[7] FAO/Food and Nutrition Division, NWFPs in nutrition. In NWFPs for Sustainable Forest Management, Yogyakarta Indonesia. NWFPs (3). FAO, Rome 1995a

[8] Department of Forestry, Nepal (DOF), Community Forest Resource Inventory Guidelines, 2061, Community Forest Division, Department of Forest, Kathmandu, Nepal 2004

[9] G.J. Osemeobo, The Non-wood Forest Products of Nigeria. In: Data Collection and Analysis for Sustainable Forest Management in ACP Countries-Linking National and International Efforts. EC-FAO Partnership Programme 1999. 
Available at http//www. fao.org/forestry

[10] L. J. T White, and J. F. Oates.. New data on the history of the plateau forest of Okomu,southern Nigeria: an insight into how human disturbance has shaped the African rain forest 1999. Global Ecology and Biogeography Letters 8: 355-361

[11] J. A. Sayer, C. S. Harcourt, and N. M. Collins, The Conservation Atlas of Tropical Forests: Africa 1992. IUCN and Simon \& Schuster, Cambridge

[12] FORMECU, The Assessment of Land Use changes in Nigeria between 1976/78 and 1993/95: Federal Department of Forestry 1998. 117pp

[13] S.O. Bada, Community Participation in the Management of Omo Forest Reserve. Final Report for FORMECU, Federal Department of Forestry, Abuja, Nigeria 1999

[14] D.U.U. Okali, and B.A. Ola-Adams, Tree population changes in treated rainforest at Omo Forest Reserve, Nigeria 1987. Journal of Tropical Ecology 3: 291-313

[15] G.I.B. Obioho, Ecological ethnobotany and the Management of Omo Biosphere Reserve, Nigeria. In: Sustainable Forest Management in Nigeria: Lessons and Prospects (eds., L. Popoola, P. Mfon and P.I. Oni). Proceedings of the $30^{\text {th }}$ Annual Conference of the Forestry Association of Nigeria, held in Kaduna, Kaduna State.7-1 ${ }^{\text {th }}$ November, 2005. Pp 86-91

[16] A.A. Adebisi, A case study of Garcinia kola nut production-to-consumption system in $\mathrm{J} 4$ area of Omo forest reserve, South-west Nigeria. In: SUNDERLAND, T. and NDOYE, O. (eds.) Forest products, livelihoods and conservation: case studies of non-timber forest product systems, 2 (2004) 115-132

[17] S.O. Jimoh, A multiple use planning model for tropical rain forests: The case of Sasha Forest Reserve, Nigeria. Ph.D. Thesis submitted to the Department of Forest Resources Management, University of Ibadan, Nigeria 2002. 254p

[18] P. R. O. Kio, Stand development in naturally regenerated forest in S.W Nigeria. Ph.D. Thesis University of Ibadan, Ibadan 1978

[19] K. Diaw, D. Blay, and C. Adu-Anning, Socio-economic survey of forest fringe communities: Krokosua Hills Forest Reserve. A report submitted to the Forestry Commission of Ghana 2002

[20] Statistical Package for Social Sciences. SPSS for Windows,
Evaluation version. 15.0. 2006. Chicago: SPSS Inc

[21] T.O. Amusa, S.O. Jimoh, P. Aridanzi and M. Haruna, Ethnobotany and Conservation of Plant Resources of Kainji Lake National Park, Nigeria. Ethnobotany Research \& Applications. 8 (2010) $181-194$

[22] B. Chikamai, M. Tchatat, C. T. Julius and O. Ndoye, Forest Management for Non-Wood Forest Products and Services in Sub-Saharan Africa. Discov. Innov. 2009; 21(SFM Special Edition No.1). Pp 50-59.

[23] B.A. Ola-adams, Germplasm conservation in forestry. Invited paper presented at the 13th Annual Conference on Genetic Resources of Nigeria, 10-14 February 1986. FRIN, Ibadan.

[24] Etkin, and P. J. Ross, Pharmacological Implication of Wild Plants in Hausa Diet. Tucson University of Arizona Press. 1994. pp85-101

[25] J.C. Okafor, F.I. Omorodion, and P.S. Amaza, Non-Timber Forest Products, Nigeria. Technical Report Prepared for FORMECU, Ibadan Nigeria 1994.

[26] J.E.M. Arnold, Socio-economic benefits and issues in non-wood forest products use. Report of the International Expert Consultation on Non-wood Forest Products. Food and Agriculture Organization of the United Nations, Rome, Italy 1995.

[27] A.O. Isichei, Omo Biosphere Reserve, Current Status, Utilization of Biological Resources and Sustainable Management (Nigeria). Working Papers of the South-South Cooperation Programme on Environmentally Sound Socio-Economic Development in the Humid Tropics. UNESCO, Paris 1995. 52p.

[28] L. Popoola, Prevalence of, and Conservation Strategies for Non-timber forest Products in the Sudano-Sahelian zone of Nigeria. Nigerian Journal of Ecology. Vol. 4 (1), 2002 Pp. 24 -33 .

[29] B.P. Subedi, Utilization of Non-Timber Forest Products: Issues and Strategies for Environmental Conservation \& EconomicDevelopment. Workshop Theme Paper for the Workshop on the Utilization of NTFPs for Environmental Conservation and Economic Development in Nepal March 29, 1997 Asia Network for Small Scale Agricultural Bioresources. Kathmandu, Nepal. 13p

[30] FAO, Non-wood forest products for rural income and sustainable forestry. Non-wood Forest Products 7. Rome $1995 b$ 